\title{
Corridor Lamp Control System Based on Solar and LED Lighting
}

\author{
Xiangrong Ding ${ }^{1, a}$, Ping Jia ${ }^{2, b}$ \\ ${ }^{1,2}$ Guangdong Industry Technical College,Guangzhou 510300,Guangdong,China \\ adingxiangrong1965@163.com, bping.jia@163.com
}

Keywords: solar energy, LED, smart home, color temperature, anti-theft device.

\begin{abstract}
Porch lamp control system is a very important part of the smart home system. The traditional light source control system is unable to control and adjust the color temperature of the light source. This design uses LED and solar porch lamp as the light source with STC15 Series MCU intelligent control unit and wireless sensor. Compared to other similar systems, this proposed control system is more humane and intelligent control system with multiple functions mode. The control system can automatically perceive indoor brightness and human information, turn off or adjust the gallery lights, and automatically detect the owner getting up at night and start the porch lamp. Porch lamp has gradually functions, which the gradual process, brightness, and color temperature are adjustable. The porch lamp control system can analog someone at home when he go out actually, and alarm the illegal going home.
\end{abstract}

\section{Introduction}

System design inspiration comes from life. Traditional porch lamp is controlled by switch with traditional light source, which is less controllable and inconvenient. Getting up at night, we need to find a switch in the dark. Lights up the moment would cause glare. The lights suddenly on will affect the rest of others. If the porch lamp lights at night, it wastes energy, and does not comply with the requirements of environmental sustainability, light-sensitive people. At the same time it affects the quality of sleep. In the design of the smart home system, the porch light as auxiliary light source of lighting at night, does not often take seriously. In fact, the porch lamp system acts as an important role in the home life. It can light up at night, especially to the elderly, with the great convenience of living and security at night. Compared to traditional light, LED lighting has energysaving, environmental protection, long life and other advantages. Again, in the production process, different colors, different color temperature of LED chip, integrated to adjust the color and the white color temperature laid a theoretical foundation, can be produced for the provision of better lighting quality and improved lighting visual effects. The design is based on the principle of green energy, energy saving and user-friendly design[1]. The design of intelligent porch lamp control system can automatically detect the environmental brightness automatically and human information. Furthermore, the porch lamp control system has gradually the brightness and color temperature, anti-theft and other functions as follows:

(1) Power supply mode: It achieves automatic switching between solar power and commercial power. It usually uses solar power, and automatically switches the power supply to commercial power when the weather is bad or the solar battery charge level is low, so as to ensure uninterrupted power supply;

(2) Ordinary working mode: The system is in standby mode in the daytime (enough brightness), and starts automatically at night (insufficient brightness), and the power consumption of the system is very low; when it is dark or the brightness is not enough, the corridor lamps turn on automatically when the resident approaches, and automatically turns off when the resident walks away; meanwhile, the brightness and color temperature of the corridor lamps are adjustable;

(3) Night mode: The system automatically turns on the corridor lamps when the resident gets up at night, and automatically turn off after a certain delay time; moreover, gradual dimming mode is adopted; 
(4) Anti-burglary mode: In the anti-burglary mode, the system turns on the lamps of different combinations at night irregularly, so as to simulate the situation that the resident stays at home; when someone intrudes illegally, the system gives an alarm in the daytime, and gives an alarm and turns on all the corridor lamps at night, so as to scare away the illegal intruder;

(5) Back-up power mode: When the lamps fail, you can start the corridor lamps as back-up, and in this situation, the corridor lamps illuminate in the brightest mode;

(6) Mode setting: You can use the control panel buttons or wireless remote controller to set the working mode of the system.

\section{System Solution}

(1) Lamp selection

LED lamps are selected. The reasons are as follows: Firstly, LED lamps are featured by energy saving, environmental protection, high optical efficiency and long life; there is no need to turn on the corridor lamps continuously, and the corridor lamps only turn on when the resident stays at home and the environmental brightness is insufficient; moreover, green energy - solar power can be used; secondly, LED lighting brightness and color temperature can be controlled, so as to ensure the comfort of LED lighting; thirdly, it conforms to the national policy on green and environmentfriendly lighting and LED lighting[2,3].

(2) Selection of control chip

STC15F2K60S2 SCM developed by Shenzhen Hongjing Science and Technology Co., Ltd. is used as the control chip of the system, and there are three reasons for this selection: Firstly, STC series SCM has the ISP/IAP (in-system programming and in-application programming) functions, which facilitate the downloading of user programs for future expansion, commissioning and online software upgrades; secondly, STC15F2K60S2 SCM has the AD conversion module, PWM output module and a number of I/O ports integrated, which meets the application requirements of the system, effectively simplifies the peripheral interface circuit and is cost-effective; thirdly, STC SCM is featured by different modes (low-speed mode, sleep mode and standby mode), low power consumption and high watchdog reliability, which further reduce the power consumption and increase the reliability of the system; fourthly, STC SCM is a mature enhanced 8051 SCM and guaranteed on technical support and goods supply[4].

(3) Power solution

LED lamps are used as corridor lamps, so the power consumption is relatively low. Considering energy saving, environmental protection and practical application, the system design adopts the dual power input solution, one is solar power input and the other one is commercial power input. Solar power and LED lighting are a perfect combination, conforming to the national regulations on the development of energy-saving, environment-friendly and green energy sources and light sources. Usually, the system uses solar power, and automatically switches the power supply to commercial power when the weather is bad or the solar battery charge level is insufficient, and switches the power supply back to solar power when the solar battery charge is restored[1].

The block diagram of the corridor lamp control system based on solar and LED lighting is shown in Fig. 1.

\section{Hardware Design}

(1) SCM minimum system and on-line programming design

STC15F2K60S2 SCM is a 1T (1 machine cycle is only one clock cycle) single-chip microcontroller, featured by fast operation and rich internal resources, including a 60KB flash program memory, a 2048-byte SRAM, a 8-channel high-speed 10-bit AD converter, a 3-channel PWM output channel and up to $38 \mathrm{I} / \mathrm{O}$ ports. In addition, STC15F2K60S2 SCM integrates an internal clock and a reset circuit, which can run the program upon power connection. Therefore, 
STC15F2K60S2 SCM itself is a SCM minimum system without the need for the connection of a peripheral circuit[5].

The in-system programming (ISP) of the STC15F2K60S2 SCM achieves data communication through the SCM UART, and generally, the MAX232 or STC232 conversion chip is used to achieve the level conversion between PC RS-232 serial interface and SCM serial interface; however, considering that the RS-232 serial interface is not a PC's standard configuration, and more and more PCs and laptops use a unified USB serial communication interface instead of an RS-232 serial interface, so the system uses the CH340T as the conversion chip to achieve the level conversion between STC15F2K60S2 SCM interface and PC USB interface, so as to constitute the in-system programming system. It should be noted that the in-system programming software of STC series SCM uses the RS-232 serial interface for data communication, and when the USB communication interface is used, a dedicated USB driver has to be run (in-system programming software and USB driver are available for download at the STC SCM official website) to generate a virtual serial port number, and this serial port number will be used for data communication at the time of in-system programming. The in-system programming circuit of STC15F2K60S2 SCM not only facilitates the downloading of user programs for application expansion, but also achieves future online software upgrades[4,10-12].

(2) Automatic solar power and commercial power switching module

One of the features of the system is the automatic switching between solar power (equipped with a $12 \mathrm{~V}$ battery) and $12 \mathrm{~V}$ commercial power converted by an adapter. When the solar battery voltage drops to $10.8 \mathrm{~V}$, the SCM detects that the voltage input of the solar battery is less than the preset value, and then it outputs the control signal and automatically switches the power supply of the system to commercial power. When the

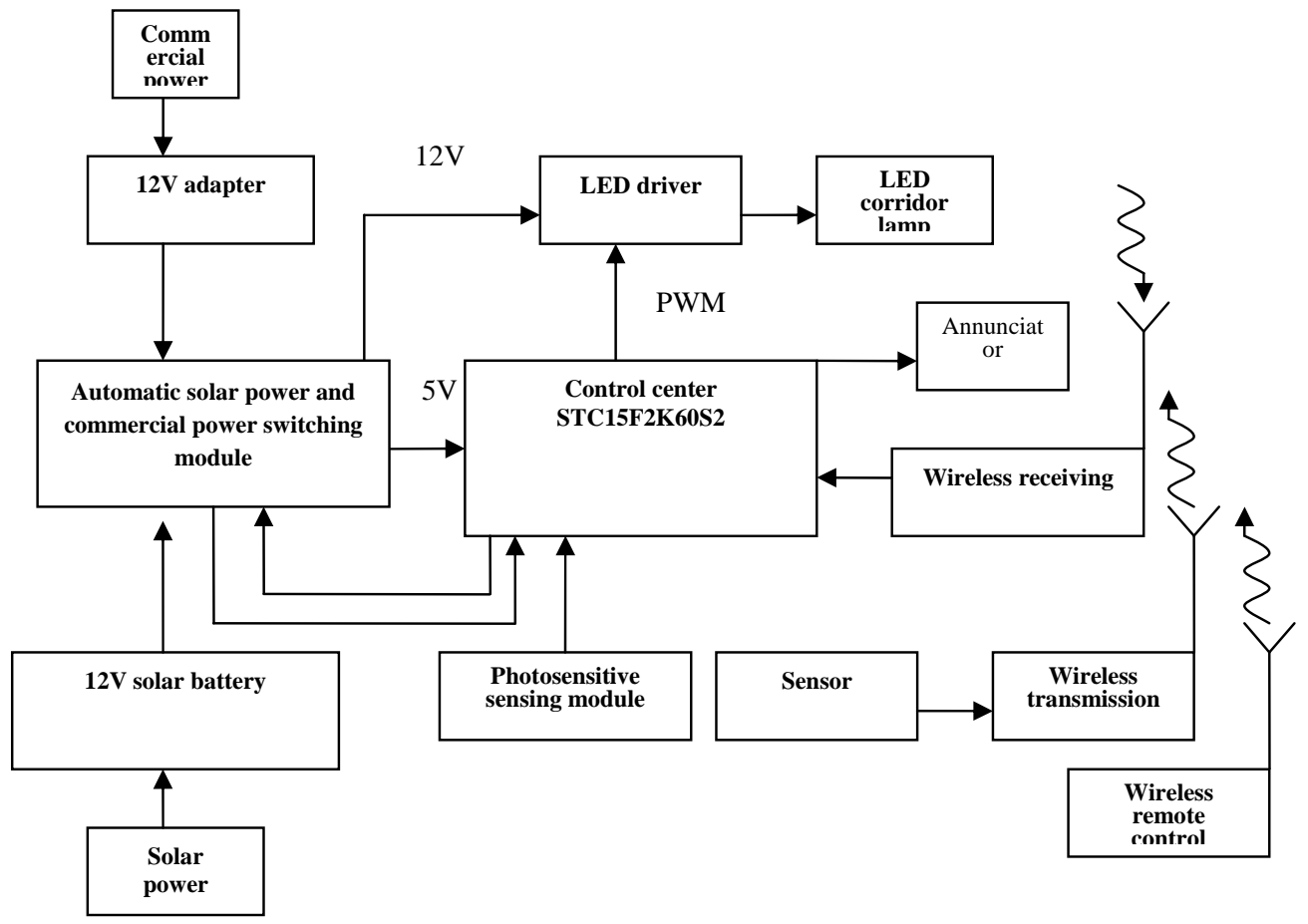

Fig.1. System block diagram.

battery voltage is restored to $12 \mathrm{~V}$, the SCM outputs the control signal to switch the power supply back to solar power. If sufficient, the solar power will be used to meet the green energy requirements; if insufficient, the commercial power will be used to ensure the power supply continuity of the system. When the system uses solar power, the voltage of the solar battery varies 
from $10 \mathrm{~V}$ to $14.5 \mathrm{~V}$ approximately, and the system control center uses the IC-LM2576 to convert the power supply from $12 \mathrm{~V}$ to $5 \mathrm{~V}$. LM2567 is featured by stable voltage, low heat generation and high efficiency[6].

(3) LED driver and LED light mixing system

LED corridor lamps are composed of high color temperature $(6000 \mathrm{~K})$ and low color temperature (3000K) LED lamps, and MOSFET IRF740 is used to achieve constant current driving. IRF740 is a voltage-controlled current device, which is controlled using the pulse width modulation (PWM) method. The current is controlled through changing the duty cycle of the voltage, so as to achieve the adjustment of brightness and color temperature of the LED lamps, and the dimming adjustment ranges from extinguishment to maximum brightness.

Color temperature adjustment of the LED corridor lamps is achieved through different PWM duty cycles of the high and low color temperature LED drivers, and such adjustment can improve the comfort of LED lighting. Under different circumstances (temperature, humidity) and mood conditions, people have different lighting requirements, mainly on color temperature and brightness.

Dimming is achieved through increase or decrease in the PWM duty cycles of the high and low color temperature LED drivers at the same ratio.

(4) Photosensitive module

The photosensitive module is used for the detection of environmental brightness, so that the system is in standby mode in the daytime, and starts only at dark (or in the case of insufficient brightness). In this way, the working time of the system is reduced, so as to extend its life and minimize its power consumption.

(5) Wireless sensing module

The wireless sensing module is used to detect whether the resident gets up at night when the corridor lamps are set to the night mode, and the lamps turn on automatically if the module detects that the resident gets up at night. The wireless sensing module can adopt the infrared sensing technology, and the sensor can be installed beside the bed. When the resident sits up, the infrared sensor can sense the infrared signal, which is transmitted to the system through the wireless transmission module, and then the system gives instructions automatically to turn on or off the corridor lamps[7,8].

(6) Wireless transmission and receiving module, and wireless sensing module

The wireless transmission and receiving module is an off-the-shelf paired module. The encodable wireless receiving module uses an 8-bit address code and a 4-bit data code, and there are 6561 address codes. Only when the address code of the receiving module is consistent with that of the transmitting module, the receiving module can output a valid signal, so that there is no need to worry about the problem of crosstalk; moreover, there are 15 data codes for data receiving, so as to distinguish between the different control functions[9].

The wireless transmitting and receiving module is used for wireless sensing, so as to achieve signal transmission and wireless remote control over system functions.

\section{Software Design}

According to the system functions, the smart corridor lamp control system software includes main program, wireless input data detection subprogram, ordinary working mode subprogram, night mode, anti-burglary mode, anti-burglary function subprogram, man-machine interaction subprogram, etc. Fig. 2 is the flowchart of the main program of the system.

\section{Conclusion}

Gallery Light Control System, a set of solar-powered LED green lighting with intelligent control in one system, has design features of green energy-saving, low-carbon environmental protection and user-friendly features.

This design can solve the LED lighting brightness, color temperature control and regulation by adjusting LED brightness, color temperature, and can effectively create a suitable in getting up at 
night, walking at night lighting environment. The same time, the concept can be extended to other areas of LED lighting, such as the home LED trunk lighting system. The system have an obvious advantages of LED lighting, not only in energy saving, environmental protection, but also in creating a comfortable lighting environment.

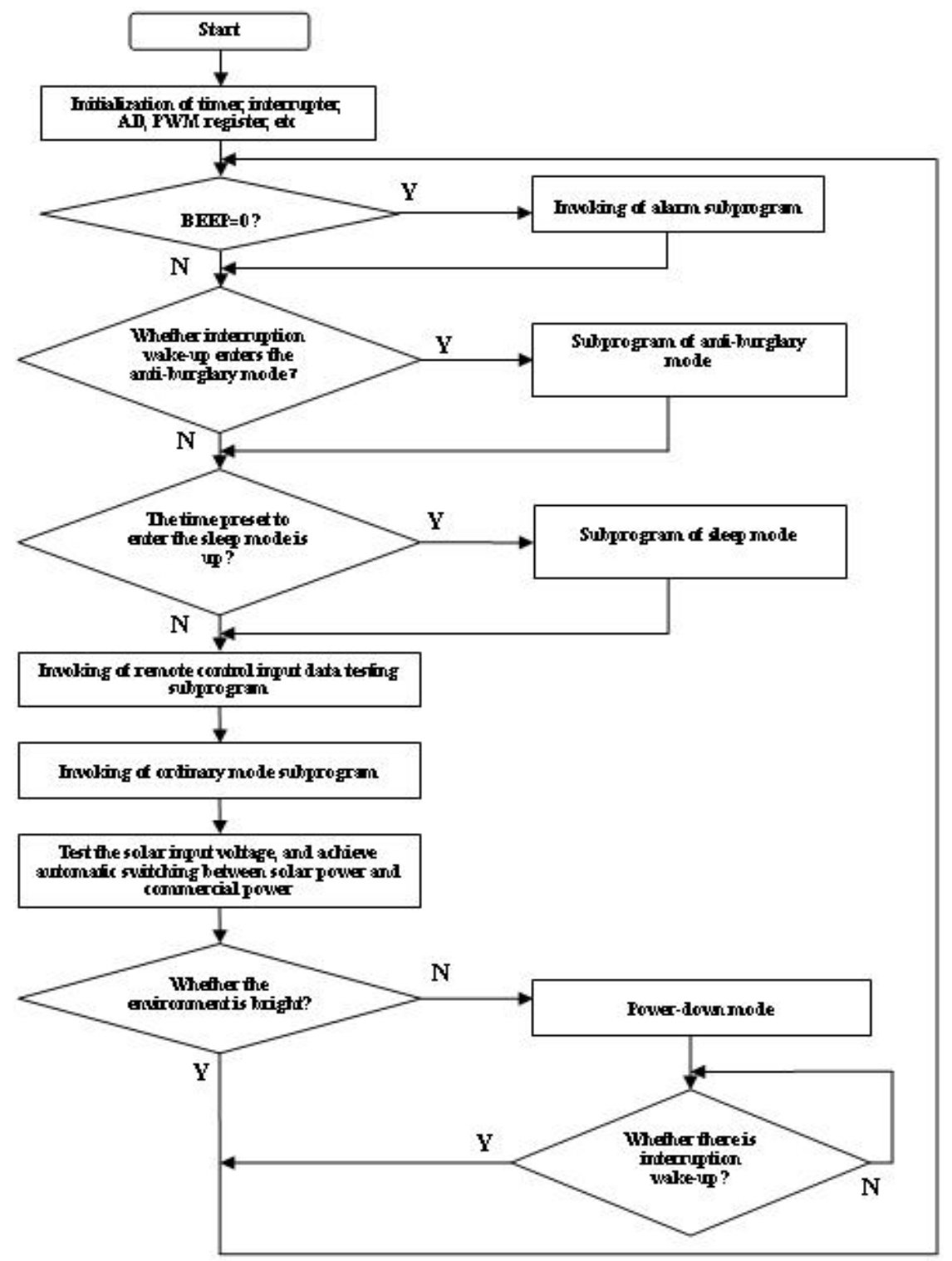

Fig.2. Program flowchart of smart corridor lamp control system

The system can automatically detect ambient lighting and determine whether you need to light on or off. Furthermore, the system can solve the problem of exploring switch at night, can protect human eye by gradually light up and down. Brightness and color temperature of LED porch lamp according to the needs of the owner are free to set by the master. Porch lamp system can simulate the owner light up at home.

The entire porch lamp system is concision, cost-effective, practical, flexible, easy to expand, and could be further developed with smart home system and home overall LED lighting system. 


\section{References}

[1] Hailong P., Quanming L., Lisha L., et al. LED Tunnel Lighting intelligent control system.Measurement \& Control Technology.-2011.-30,8.-P.63-66.

[2] Zhimin Z., Jihai Z., Aihua J..LED Drive Circuit Design Examples.Electronic Industry Press.2008.-P.108-112.

[3] Zuming L.. Explanation of LED Lighting Driver Design Cases.Chemical Industry Press.-2011.P.110-115.

[4] Xiangrong, D. .Monolithic Microcomputer Principle and Interface Technology. Electronic Industry Press.-2012.-P.228-232.

[5] Juan Z.. Programming Method of LCD Display Interface in Embedded System.Techniques of Automation and Applications.-2001.-3.P.93-96.

[6] Xiaoping Z. , Yan L. , Wei L.. Design of Wireless Temperature Control System Based on Fuzzy Control.Automation Technology and Application.-2001.-3.P.23-25.

[7] Youfeng Y., Jiangdong Z., Shiping Hao. Design of Environment Monitoring System Based on SCM Technology.Measurement \& Control Technology.-2012.-31,1.-P.58-59.

[8] Yongyi M., Hongjun Z., Hongliang W.. Design of a New Embedded Wireless Sensor Network[J].Measurement \& Control Technology.-2012.-31,10.-P33-34.

[9] Chuanming Y., Pengfei S., Shengli X. , et al. Development of Projectile Speed Measurement Based on STC Microcontroller.Measurement \& Control Technology.-2012.-31,10.-P.72-75.

[10]Xiangrong D., Ping J.. Microcomputer Application System and Its Development Technology.Tsinghua University Press.-2009.-P.201-211

[11] Miller W, Buys L.. Anatomy of a sub-tropical Positive Energy Home (PEH). Solar Energy, 2012, 86(1): 231-241.

[12]Young R A.. Environmental Factors.Stewardship of the Built Environment. Island Press/Center for Resource Economics, 2012: 79-136. 